\title{
Research on the Design and Implementation of Smart Home Network System based on the Embedded Technology
}

\author{
LIN $\operatorname{Nan}^{1, a}$ \\ ${ }^{1}$ College of Software Technology, Zheng Zhou University, Henan Zhengzhou, 450002, China \\ a linnan@zzu.edu.cn
}

Keywords: Smart Home; Network System; Embedded Technology; Systematic Design.

\begin{abstract}
In this paper, we design and implement the smart home network system based on the embedded technology. In the smart home system, wireless network technology was applied to the family network has become an irresistible trend. Household system based on network is the connection of all kinds of information appliances in the family and family services of various types of bridges and the home gateway is the core of the smart home system based on network. The verification and simulation shows the feasibility of the proposed implementation.
\end{abstract}

\section{Introduction}

In times of the Internet of Things where more and more everyday objects are becoming intelligent, smart homes exist no longer only theoretically, they are available for everyone. In the smart home system, wireless network technology was applied to the family network has become an irresistible trend. Not just because the wireless network can provide greater flexibility and mobility but also save and effort spent on integrated wiring fees and more because it conforms to the home network communication characteristics. With the further development of the wireless network technology, it will greatly promote the progress of the intelligent home network. Smart home is intended to study how to make use of computer and network communication technology for residential intelligent control and management in order to improve the living environment of comfort, convenience, efficiency and safety. In general intelligent household system has family control and home entertainment services and home security protection function three aspects. The emergence of the Internet triggered the fourth industrial revolution and the human beings entered the information age. Along with the computer, communications, automatic control, microelectronics, such as the development of technology, home network with the help of existing computer network technology and communication technology which will be all kinds of home appliances and household equipment networking which provide diversification, personalization, and for people through the network security and efficient service. Traditional Internet applications on PC (personal computer) are turning to today's Internet application on embedded devices which means that we entered the era of embedded Internet. According to experts predict that in the transmission of the information on the Internet in the future, there will be $70 \%$ of the information from the small embedded systems [1-2].

Evolution in ICT led to the emergence of smart homes which offer new opportunities to improve people's in-home comfort by providing increased communication, awareness, and functionality. To the best of our knowledge, few studies utilized the behavior pattern to detect abnormal user behavior for target of energy saving. Previous researches of usage patterns mainly focus on the feature of energy disaggregation. Also known as the home network device information home appliances, electronic equipment are a kind of intelligence and it is the traditional household electronics product of networking. The process of information and intelligent can quickly and easily access to the Internet like a PC and remote access, monitoring and surveillance. Household system based on network is the connection of all kinds of information appliances in the family and family services of various types of bridges and the home gateway is the core of the smart home system based on network. Its function is: in the family, the family gateway through local area network (LAN) connects all sorts of equipment within the family. In essence, home gateway is a combination of domestic modem and router and the 
introduction of new service dynamic transfer function and the remote management of the system as well as the upgrade. In fact, adding an embedded server can amend any broadband network terminal device to family gateway. As far as the smart home system based on Internet is concerned, how to intelligent home appliances and the Internet connection is a key to solve the problem which is also a research hotspot in recent years [3]. The mode of connection is normally finalized by which contains embedded web services gateway connected to the Internet. This kind of structure is the core of the desktop computer or high-performance embedded processors as a gateway which support the TCP/IP protocol stack and run Internet service program. The user through a web browser to access gateway implementation running on the server for remote access and control of these embedded devices. So as to realize embedded devices connected to the Internet [4]. Therefore, to optimize the current pattern of the smart home's network connection, we conduct research in this paper on the item.

\section{The Proposed System}

The Overview of the Network Connection. In grid computing environment, due to the grid services are provided by different grid server and different grid server provides various grid service. Therefore, in the model described in a store with a grid server provided by the grid service function with each storage unit to show the server provides a grid service is very intuitive. The general socket programming for the network interface is shown in the following sample code blocks.

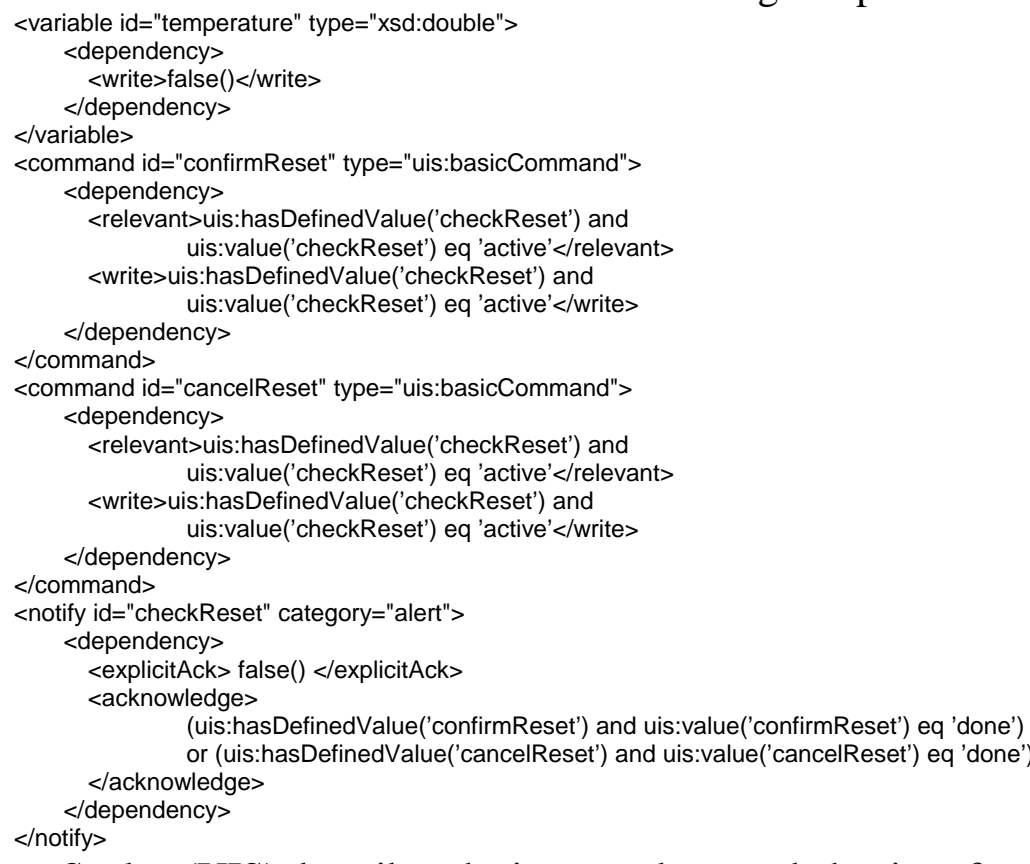

The User Interface Socket (UIS) describes the input and output behavior of a specific target device on an abstract level and contains a flat set of socket elements that provide a synchronized communication channel to the controlled device and its current state. This model is mainly used to solve the embedded gateway IP when connected to the Internet problem caused by limited resources. With the gradually development of information, community network is more and more popular also more and more mature. Therefore, we can make the community in the network server acts as the proxy server functions of the new model and it can be regarded as the pattern on the implementation of a more viable option. If the family gateway IP is not fixed which is changing every time connected to the Internet, this means that the user login from Internet network gateway must know the IP address of the gateway. So, the user must remember that every time a different IP address. The following sections will introduce the corresponding techniques in detail for solution.

The System Implementation. Network technology has the benefit of with the low power consumption, low cost, short time delay, security, network characteristics of large capacity and flexible working band. The physical layer and MAC layer communication protocol to follow the IEEE standards which can use global unity without application spectrum. Wireless smart home 
system management center to Internet home wireless router to the network communication between the people mainly adopts WLAN wireless technology to connect to the network. Management center to the users of mobile phone alarm or control information through GSM/GPRS network and it is using the mobile communication company business network. Various hardware composition of the different communication network channels, both indoor all sorts of video, audio, data and other information between various detectors and detector close between control center and information transmission channel and also including indoor all sorts of information transmission between mobile phone or computer with users or control. The user terminal mainly refers to the mobile phone and computer. When users go out at ordinary times, mobile phone is usually in a working on standby at any time and the alarm information will be the first to arrive at a mobile phone. Mobile phone users once received information coming from the home alarm center which can control the control points in the home at any time by phone. If you want to know more detail about the home situation, then you can open the side of the computer or $3 G$ video phones through the network video monitoring in the home of everything to access the further processing. The figure 1 shows the system description.

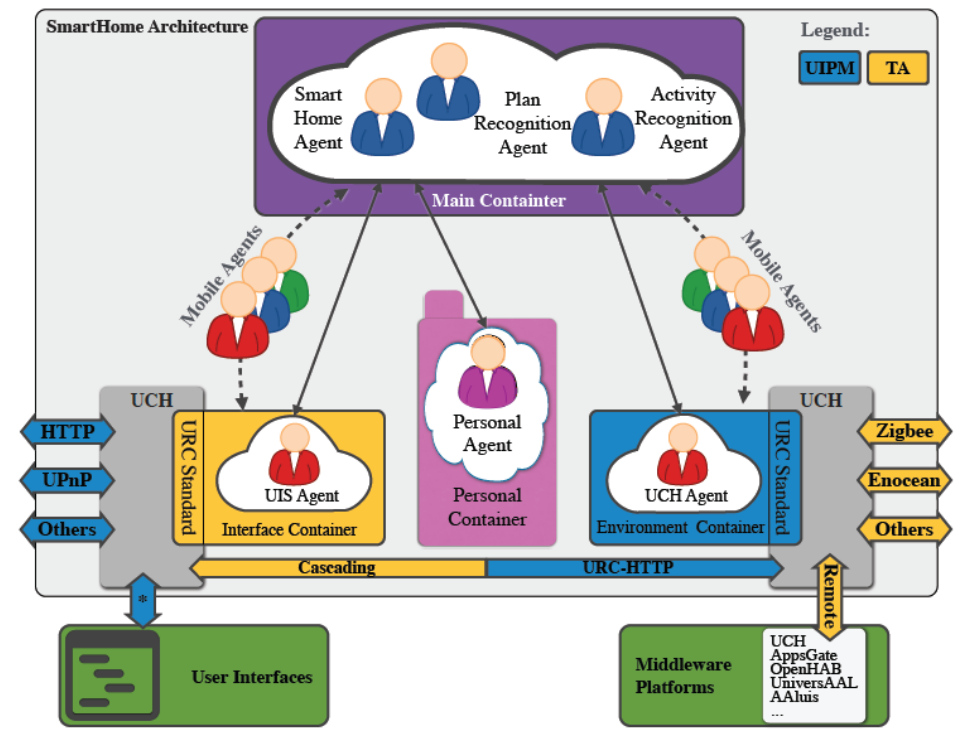

Fig. 1The Structure of Our Designed System

The Hardware Description of the System. Our system is implemented through the following hardware devices. The sensing unit is constructed by combined means of wireless sensors and video. Video detector is made up of analog cameras and video control center of a processing unit. Conventional sensors such as gas leak detection sensor and infrared sensor has a mature circuit. This scheme only needs to be sensing part module combination can be made wireless sensor. In wireless network wireless sensor as a terminal node or routing module which induced the information as well as other routing nodes and wireless module communication also can communicate with control center network coordinator. The actual when using, need to increase the wireless network adapter, through the software configuration, can with the user home wireless router connected to the Internet. . Under normal circumstances, the user's network is pasta, wireless router non-stop work, in this way, the head of the household outside surveillance video at home can access at any time by the computer, at the same time can also through the network of the remote control to adjust video probe bracket and the camera focal length, aperture, etc. However, the research and development in this area seems to drift in two branches: the middleware platforms and the intelligent services. On the one hand, the middleware platforms are concerned with integration and usability of smart home appliances. One of their main tasks is to adapt these targets and provide unified interfaces to users and/or applications. GSM module adopts both GPRS function SIM300C as the main chip, with convenient function extension in the future. MSP430 controller can be used in the wireless module already and also can be used as a controller. The GSM module in meet the design function on the basis of can reduce the hardware cost. MCU with SIM300C can be directly through the UART communication, but for the sake of their respective and computer on-line debugging convenience, practical circuit with serial 
interface conversion circuit, and using the jumper wire to choose connection. Based on the analysis, they should trigger some actions to support the residents. Such intelligent smart home services are usually agent-based approaches based on a multi-agent system. The next section will illustrate the verification and simulation of our proposed system.

\section{Experiment and Verification}

In this section, we conduct numerical simulation on the proposed and designed system. By the central control of fixed equipment management system issued by increasing instruction code instructions, MCU and a serial port sent to the control center by computer and sent via two line interface to the coordinator. Once again by the coordinator to send to the terminal nodes, the terminal node receives the complete data via a serial port sent to the PC again. Finally, in the PC to complete the terminal node to receive data compared with the control center of data sent in. The following figure 2 illustrate the structure and logistic for the verification procedure.

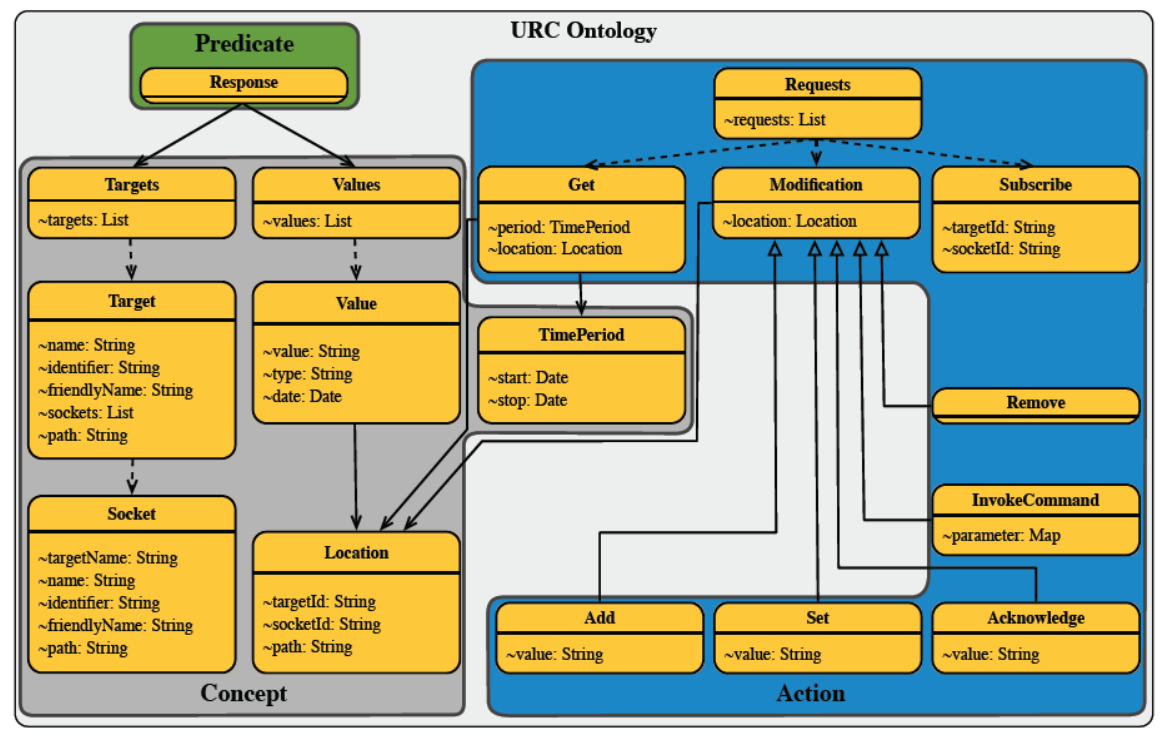

Fig. 2The Simulation and Verification of the System

\section{Conclusion and Summary}

The development of modern science and technology, especially with the rapid development of computer technology and network technology, not only changed the way we work, and gradually changed our way of life. Requirements, automatic and intelligent office cannot only, also need household life gradually became more automatic and intelligent. Smart home is in this context. Smart home is a blend of computer, network, automatic control, sensing and other technology, and related to ecology, environment, energy and other fields of comprehensive systematic engineering. In addition to meet the requirements of automatic control, but also to achieve energy saving, environmental protection, human, and personalization. With the accelerating rhythm of life, people want to have a more comfortable and more comfortable modern household, to its security, intelligent, economy put forward higher requirements. Control, communication, computer, and the development of network technology, especially the wide application of embedded type system, promoted the rapid development of information appliances, network technology and main equipment of home network is no longer a PC, but embedded type system of intelligent home network control platform, its internal and external network connection for household and internal network connection information home appliances and equipment provides a basic platform.

A sort of smart home system is designed and completed which based on Embedded System, IP, GSM/GPRS/3G and other wireless communication technologies. The system composition of the proposed is analyzed from the hardware design, network applications, software design and other 
respects. In general intelligent household system has family control and home entertainment services and home security protection function three aspects. The emergence of the Internet triggered the fourth industrial revolution and the human beings entered the information age. The verification section proves that the proposed system is acceptable.

\section{References}

[1] Jiang X, Dawson-Haggerty S, Dutta P, et al. Design and implementation of a high-fidelity ac metering network[C]//Information Processing in Sensor Networks, 2009. IPSN 2009. International Conference on. IEEE, 2009: 253-264. [1]

[2] Xin-chang Z, Feng-quan Z, Corporation X G. Smart grid leads the journey to innovative smart home and energy consumption patterns[J]. Power System Protection \& Control, 2014.

[3] Ye Z, Li Y, Zhao Q, et al. A Falling Detection System with wireless sensor for the Elderly People Based on Ergnomics[J]. International Journal of Smart Home, 2014.

[4] Hong J, Suh E, Kim S J. Context-aware systems: A literature review and classification[J]. Expert Systems with Applications, 2009, 36(4): 8509-8522.

[5] Zhou S, Wu Z, Zhang J L X. Real-time Energy Control Approach for Smart Home Energy Management System[J]. Electric Power Components \& Systems, 2014, volume 42(3):315-326(12).

[6] Niyato D, Xiao L, Wang P. Machine-to-machine communications for home energy management system in smart grid[J]. Communications Magazine, IEEE, 2011, 49(4): 53-59.

[7] Obaid T. ZIGBEE BASED VOICE CONTROLLED WIRELESS SMART HOME SYSTEM[J]. International Journal of Wireless \& Mobile Networks, 2014, (1):47-59.

[8] Han D M, Lim J H. Smart home energy management system using IEEE 802.15. 4 and zigbee[J]. Consumer Electronics, IEEE Transactions on, 2010, 56(3): 1403-1410. 